Editorial

\title{
Special Issue on Advanced Biometrics with Deep Learning
}

\author{
Andrew Beng Jin Teoh ${ }^{1, *(D)}$ and Lu Leng ${ }^{2}$ \\ 1 School of Electrical and Electronic Engineering, College of Engineering, Yonsei University, \\ Seoul 120749, Korea \\ 2 School of Software, Nanchang Hangkong University, Nanchang 330063, China; leng@nchu.edu.cn \\ * Correspondence: bjteoh@yonsei.ac.kr
}

Received: 16 June 2020; Accepted: 24 June 2020; Published: 28 June 2020

\section{Introduction}

Biometrics, such as fingerprint, iris, face, hand print, hand vein, speech and gait recognition, etc., as a means of identity management has become commonplace nowadays for various applications. Traditional authentication methods, including possession-based and knowledge-based methods, typically suffer from various problems. It is probable that possessions, such as an ID card or key, can be stolen, broken, or lost; while it is probable that knowledge, such as a password or PIN, can be forgotten or guessed. Compared with the traditional authentication methods, biometric recognition methods are more convenient and secure [1].

Biometric systems follow a typical pipeline that is composed of separate acquisition, preprocessing, feature extraction and classification. Deep learning as a data-driven representation learning approach has been shown to be a promising alternative to conventional data-agnostic and handcrafted pre-processing and feature extraction for biometric systems. Furthermore, deep learning offers an end-to-end learning paradigm to unify preprocessing, feature extraction and recognition based solely on biometric data [2]. The main advantages of deep learning include a strong learning ability, wide coverage, good adaptability, data-driven, good transferability, etc.

\section{Advanced Biometrics with Deep Learning}

In light of the above, this Special Issue collected high-quality, state-of-the-art research papers that deal with challenging issues in advanced biometric systems based on deep learning. A total of 32 papers were submitted, 12 of which were accepted and published (i.e., 37.5\% acceptance rate). The 12 papers can be briefly divided into 4 categories as follows according to biometric modality.

\subsection{Face}

The paper, authored by H. Tu, G. Duoji, Q. Zhao and S. Wu, extracted invariant features from a single sample per subject for face recognition. The authors generated additional samples to enrich the intra-variation and eliminate external factors [3]. Another paper by S. Zhou, C. Chen, G. Han and X. Hou, proposed a novel loss function, termed as double additive margin Softmax loss (DAM-Softmax) for convolutional neural networks (CNNs) in face recognition [4]. The presented loss has a clearer geometrical explanation and can produce highly discriminative features. B. Ríos-Sánchez, D. Costa-da-Silva, N. Martín-Yuste and C. Sánchez-Ávila, described and evaluated two deep learning models for face recognition in terms of accuracy and size, which were designed for the applications in mobile devices and resource saving environments [5]. The 4th paper, authored by Z. Yang, J. Li, W. Min and Q. Wang, presented real-time pre-identification and cascaded detection for tiny faces to reduce background and other irrelevant information [6]. The cascade detector consisted of a two-stage 
convolutional neuron network to detect tiny faces in a coarse-to-fine manner. The face-area candidates were pre-identified as a region of interest (ROI) based on a real-time pedestrian detector, while the set of ROI candidates was the input of the second sub-network instead of the whole image.

\subsection{Medical Electronic Signal}

Medical electronic signals referring to electrocardiogram (ECG) and electroencephalogram (EEG) signals have been identified as a type of behavioral biometrics. The paper, authored by E. Ihsanto, K. Ramli, D. Sudiana and T. S. Gunawan, focused on the accuracy and processing speed of ECG recognition [7]. They proposed a fast and accurate two-stage framework that consisted of ECG beat detection and classification. Hamilton's method and Residual Depthwise Separable CNN (RDSCNN) were used for ECG beat detection and classification, respectively. Another paper by F. Li, X. Li, F. Wang, D. Zhang, Y. Xia and F. He, aimed at enhancing the classification accuracy of P300 EEG signals in a non-invasive brain-computer interface system [8]. They employed principal component analysis (PCA) to remove the noise and artifacts in the data as well as to increase the data processing speed. Furthermore, the parallel convolution method was used for P300 classification, which increased the network depth and improved the accuracy. The third paper, authored by D. Wang, Y. Si, W. Yang, G. Zhang and T. Liu, proposed a novel method suitable for short-term ECG signal identification [9]. An improved heart-rate-free resampling strategy was employed to minimize the influence of heart-rate variability during processing. The PCA Network (PCANet) for feature extraction was implemented to determine the potential difference between subjects.

\subsection{Voice Print}

Most deep learning-based speaker variability embedding is trained in a supervised manner and requires massive labeled data. To address this issue, W. H. Kang and N. S. Kim proposed a novel technique to extract an i-vector-like feature based on the variational auto-encoder, which was trained in an unsupervised manner to obtain a latent variable, which was represented by a Gaussian mixture model distribution [10]. Another paper, authored by J. Li, X. Zhang, M. Sun, X. Zou and C. Zheng, introduced attention-based long short-term memory (LSTM) to extract representative frames for spoofing detection in noisy environments [11]. Since the selection and weighting of features can improve the discrimination [12,13], the specific and representative frame-level features were automatically selected by adjusting their weights in the framework of attention-based LSTM.

\subsection{Other Modalities}

In addition to the above three biometric modalities, the remaining three papers are based on other biometric modalities, namely periocular, person re-identification and finger-vein. The paper, authored by L. C. O. Tiong, Y. Lee and A. B. J. Teoh, studied the periocular recognition under unconstrained environments and proposed a dual-stream CNN, which employed the Orthogonal Combination-Local Binary Coded Pattern (OCLBCP) as a color-based texture descriptor [14]. Their network aggregated the RGB image and OCLBCP by using two distinct late-fusion layers. Another paper by Y. Liu, H. Yang and Q. Zhao, focused on the misalignment problem in person re-identification [15]. They presented a two-branch deep joint learning network, where the local branch generated misalignment robust representations by pooling the features around the body parts, while the global branch generated representations from a holistic view. A hierarchical feature aggregation mechanism aggregated different levels of visual patterns assigned learned optimal weights within body part regions. The third paper, authored by H. Qin and P. Wang, proposed an approach to extract robust finger-vein patterns for verification and a supervised learning scheme for vein pattern encoding [16]. Stacked CNN (SCNN) and LSTM were utilized to predict the probability of a vein pixel belonging to a vein pattern. 
The accepted papers contain the latest scientific research progress and remarkable achievements, which have important reference significance and values for the research in the fields of biometric recognition, deep learning and computer vision.

\section{Technical Challenges and Future Development Trends}

Although deep learning methods commonly outperform traditional handcrafted methods for biometric recognition, there are still several technical challenges and open problems, including the availability of high-quality labeled training samples, high computation and storage cost, hardware requirements, poor portability, complicated model design, low interpretability, etc.

Thus, the efforts of solving the aforementioned challenges compose the future trends of deep learning. Many new types of machine learning problems, such as weakly-supervised, semi-supervised and self-supervised learning have been explored to reduce the dependence on the labelled training samples. Compression technologies, such as pruning, quantization and knowledge distillation are employed to reduce the computation/storage cost. Light-weight deep learning models such as MobileNet, ShuffleNet are developed for mobile environments or to improve portability. In addition, many researchers are trying to improve the interpretability of deep learning models.

To sum up, deep learning technologies will definitely play an increasingly important role in biometric recognition with their rapid development.

Author Contributions: Writing—original draft preparation, L.L.; writing-review and editing, A.B.J.T. Both authors have read and agreed to the published version of the manuscript.

Funding: This work was supported by the National Research Foundation of Korea (NRF) grant funded by the Korea government (MSIP) (NO. NRF-2019R1A2C1003306) and National Natural Science Foundation of China (61866028).

Conflicts of Interest: The authors declare no conflict of interest.

\section{References}

1. Jain, A.K.; Nandakumar, K.; Ross, A. 50 years of biometric research: Accomplishments, challenges, and opportunities. Pattern Recognit. Lett. 2016, 79, 80-105. [CrossRef]

2. LeCun, Y.; Bengio, Y.; Hinton, G. Deep learning. Nature 2015, 521, 436-444. [CrossRef] [PubMed]

3. Tu, H.; Duoji, G.; Zhao, Q.; Wu, S. Improved Single Sample Per Person Face Recognition via Enriching Intra-Variation and Invariant Features. Appl. Sci. 2020, 10, 601. [CrossRef]

4. Zhou, S.; Chen, C.; Han, G.; Hou, X. Double Additive Margin Softmax Loss for Face Recognition. Appl. Sci. 2019, 10, 60. [CrossRef]

5. Ríos-Sánchez, B.; Costa-Da-Silva, D.; Martín-Yuste, N.; Avila, M.D.C.S. Deep Learning for Facial Recognition on Single Sample per Person Scenarios with Varied Capturing Conditions. Appl. Sci. 2019, 9, 5474. [CrossRef]

6. Yang, Z.; Li, J.; Min, W.; Wang, Q. Real-Time Pre-Identification and Cascaded Detection for Tiny Faces. Appl. Sci. 2019, 9, 4344. [CrossRef]

7. Ihsanto, E.; Ramli, K.; Sudiana, D.; Gunawan, T.S. Fast and Accurate Algorithm for ECG Authentication Using Residual Depthwise Separable Convolutional Neural Networks. Appl. Sci. 2020, 10, 3304. [CrossRef]

8. Li, F.; Li, X.; Wang, F.; Zhang, D.; Xia, Y.; He, F. A Novel P300 Classification Algorithm Based on a Principal Component Analysis-Convolutional Neural Network. Appl. Sci. 2020, 10, 1546. [CrossRef]

9. Wang, D.; Si, Y.; Yang, W.; Zhang, G.; Liu, T. A Novel Heart Rate Robust Method for Short-Term Electrocardiogram Biometric Identification. Appl. Sci. 2019, 9, 201. [CrossRef]

10. Kang, W.H.; Kim, N.S. Unsupervised Learning of Total Variability Embedding for Speaker Verification with Random Digit Strings. Appl. Sci. 2019, 9, 1597. [CrossRef]

11. Li, J.; Zhang, X.; Sun, M.; Zou, X.; Zheng, C. Attention-Based LSTM Algorithm for Audio Replay Detection in Noisy Environments. Appl. Sci. 2019, 9, 1539. [CrossRef]

12. Leng, L.; Zhang, J.; Khan, M.K.; Chen, X.; Alghathbar, K. Dynamic weighted discrimination power analysis: A novel approach for face and palmprint recognition in DCT domain International. J. Phys. Sci. 2010, 5, 2543-2554. 
13. Leng, L.; Li, M.; Kim, C.; Bi, X. Dual-source discrimination power analysis for multi-instance contactless palmprint recognition. Multimed. Tools Appl. 2015, 76, 333-354. [CrossRef]

14. Tiong, L.C.O.; Lee, Y.; Teoh, A.B.J. Periocular Recognition in the Wild: Implementation of RGB-OCLBCP Dual-Stream CNN. Appl. Sci. 2019, 9, 2709. [CrossRef]

15. Liu, Y.; Yang, H.; Zhao, Q. Hierarchical Feature Aggregation from Body Parts for Misalignment Robust Person Re-Identification. Appl. Sci. 2019, 9, 2255. [CrossRef]

16. Qin, H.; Wang, P. Finger-Vein Verification Based on LSTM Recurrent Neural Networks. Appl. Sci. 2019, 9, 1687. [CrossRef]

(C) 2020 by the authors. Licensee MDPI, Basel, Switzerland. This article is an open access article distributed under the terms and conditions of the Creative Commons Attribution (CC BY) license (http://creativecommons.org/licenses/by/4.0/). 